\title{
The Applications of Soft Computing in Embedded Medical Advisory Systems for Pervasive Health Monitoring
}

\author{
Bing-Nan LI; Ming-Chui Dong; Vai Mang I \\ Dept. of Electrical and Electronics Engineering, University of Macau, \\ Macau S.A.R., China; \\ INstituto de Engenharia de Sistemas e Computadores de Macau, Macau
}

\begin{abstract}
Based on the idea of SoC (System-on-Chip), an embedded-link, selfadaptive medical advisory system is proposed for home health monitoring, which can be merged into the embedded platform of mobile or wearable medical sensors and run independently. Meanwhile, if necessary, it will up link to health centers and self-calibrate its knowledge base. To provide effective medical advices, the methods of soft computing, including temporal fuzzy variables and weighted medical rules, are applied to the proposed medical advisory system. The elementary components, including medical knowledge base, inference machine and shell, are addressed in detail. Lastly, the way to construct such embedded-link, self-adaptive medical advisory system and integrate it into a mobile cardiovascular monitoring device is introduced too.
\end{abstract}

Keywords: medical advisory system, temporal fuzzy, weighted rules, health monitoring

\section{Introduction}

Nowadays, people pay more and more attention to their health condition. However, the frequent visiting to hospitals, mostly for routine health examination and/or medical consultation, is a heavy load for patients as well as for physicians and nurses. Hereby the research on home healthcare, especially home healthcare monitoring, is booming due to improving the quality of people's life while depressing medical expenditure. The reported research works focus on constructing the platform for home health monitoring. It collects the vital signs (Franchi et al. 1998; Jannett et al. 2002) or monitors users' daily life (Ogawa et al. 2002; Castro et al. 2001), then submits the collected data to central server for further medical advices 
via various communicating mechanisms (Nambu et al. 2000; Crumley et al. 2000).

Recently, with the prevalence of mobile apparatus and embedded systems, the research on wearable medical sensors and pervasive health monitoring is increasing popular (Diana et al. 2003). However, in terms of these systems, one of the common flaws is deficiency of on-system intelligence. For example, most of them just generate alarming messages if and only if there are some abnormal symptoms. But from the viewpoint of medicine, it is generally too late for the diseases might have become unrecoverable once the signs were abnormal. Therefore, to promote the pervasive health monitoring, it is essential to develop the home usersoriented medical advisory systems suitable for the embedded platforms. The desired system will analyze the long-term symptoms to assess users' health condition or find out the intrinsic information about the diseases' evolution.

In this paper, an embedded-link, self-adaptive medical advisory system is proposed, which can be merged into the embedded platform of mobile or wearable medical sensors and run independently. Meanwhile, if necessary, it will up link to health centers and self-calibrate its knowledge base. Since it is designed for home users with little of medical professional knowledge, the medical advisory system will be built on the legible linguistic framework. The methods of soft computing, including temporal fuzzy variables and weighted medical rules, are applied to the proposed system.

\section{Building the Medical Knowledge Base}

Most of the reported medical expert systems seem too professional to be applied in home healthcare. For example, the diagnostic program of heart diseases at MIT demands more than 100 vital parameters including the abstruse data from laboratory testing. It is useful to aid medical professionals at health centers but doomed to fail in home healthcare. As to home users with little medical knowledge, for home health monitoring, they merely care the evolution of health condition, and if any, the related medical advices in daily life. Benefited from the advanced technology of machine learning, it is feasible to elicit the medical advisory knowledge from the long-term monitoring dataset of diagnosed patients. Different from the knowledge for medical diagnosis, which is defined and described rigorously, here the elicited knowledge base comprises a series of medical rules with temporal fuzzy variables. 


\subsection{Temporal Fuzzy Variables}

Generally, the health monitoring devices collect several vital signs so to reflect the health condition of home users. Moreover, for mobility, these home-oriented devices only conserve limited times of monitoring data. Therefore, it is essential to introduce a temporal variable to reflect the long-time variation of vital signs.

Suppose the monitored vital signs are symbolized as " $V S_{1}, V S_{2} \ldots V S_{n}$ " and for each of them, in terms of the traditional mobile health monitoring devices, merely limited amount of historical data are recorded as " $V S_{i 1}$, $V S_{i 2} \ldots V S_{i m}$ " $(i \in[1, n])$ according to the time of acquisition. To reflect the accumulative medical information, a pair of temporal variables " $V S_{i}, k_{i}$ " is introduced for medical advisory system. Here " $V S_{i}$ ", defined by equation (1), synthesizes the historical records and ' $k_{i}$ ', defined by equation (2), reflects the variation.

$$
V S_{i}=\left\{\begin{array}{cl}
V S_{i 1} & \text { Initializtion } \\
\frac{V S_{i}+V S_{i}^{\text {new }}}{2} & \text { Updating }
\end{array}\right.
$$

where " $V S_{i}^{\text {new }}$ " means the newest monitoring record such as " $V S_{i 2}$ ", " $V S_{i 3}$ " $\ldots$ It is shown that " $V S_{i}$ " eventually updates as following:

$$
\begin{aligned}
V S_{i}=V S_{i 1} \rightarrow \frac{V S_{i 1}+V S_{i 2}}{2} & \rightarrow \frac{V S_{i 1}+V S_{i 2}+2 V S_{i 3}}{2^{2}} \rightarrow \frac{V S_{i 1}+V S_{i 2}+2 V S_{i 3}+2^{2} V S_{i 4} \ldots}{2^{3}} \ldots \\
& \rightarrow \frac{V S_{i 1}+V S_{i 2}+\sum_{\mathrm{j}=3}^{\mathrm{m}} 2^{(j-2)} V S_{i j}}{2^{(\mathrm{m}-1)}} \rightarrow \cdots
\end{aligned}
$$

Hence, though quite simple computation in equation (1), the variable " $V S_{i}$ " has been implicitly weighted according to its temporal sequence.

$$
k_{i}=\left\{\begin{array}{cc}
0 & \text { Initialization } \\
\text { Sign }\left|v_{i}\right|\left|\frac{\left|v_{i}\right|-\left|v_{i}^{\text {old }}\right|}{\left|v_{i}\right|+\left|v_{i}^{\text {old }}\right|}\right| & \text { Updating }
\end{array}\right.
$$

where "| |"is to extract the absolute value while "Sign ||" is to get the sign. The values " $v_{i}$ " and " $v_{i}^{\text {old }}$ " are respectively defined as follows. 


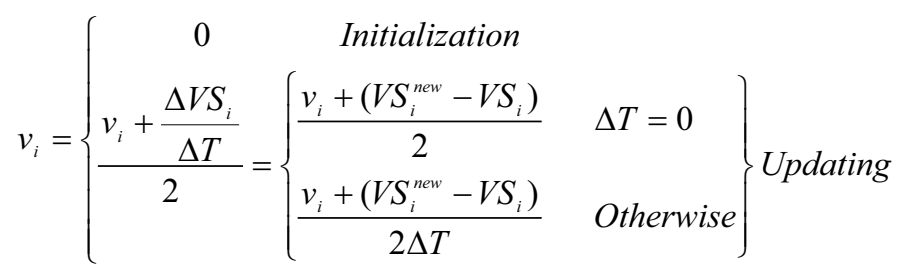

And " $v_{i}^{\text {old }}$ " is evaluated as the " $v_{i}$ " before updating. Here " $\Delta T$ " comes from the day-based monitoring interval.

In equation (3), " $k_{i}$ " has been generalized into the interval " $(-1,1)$ " so it is appropriate to fuzzify the variable " $k_{i}$ " for later fuzzy medical advisory system. According to Figure 1, the correspondent membership function of temporal fuzzy variable " $\mu_{i}$ " is defined via the following equations (Pedrycz, 1998):

$$
\begin{aligned}
& \mu_{i}^{V U}=\left\{\begin{array}{ll}
1, & x \in[0.8,1] \\
(x-0.6) / 0.2, x \in[0.6,0.8)
\end{array} \quad \mu_{i}^{M U}= \begin{cases}(0.7-x) / 0.2, & x \in[0.5,0.7] \\
(x-0.3) / 0.2, & x \in[0.3,0.5)\end{cases} \right. \\
& \mu_{i}^{U}=\left\{\begin{array}{ll}
(0.4-x) / 0.2, \quad x \in[0.2,0.4] \\
x / 0.2, \quad x \in[0,0.2)
\end{array} \quad \mu_{i}^{N}= \begin{cases}(0.2-x) / 0.2, & x \in[0,0.2] \\
(x+0.2) / 0.2, & x \in[-0.2,0)\end{cases} \right. \\
& \mu_{i}^{D}=\left\{\begin{array}{l}
-x / 0.2, \quad x \in[-0.2,-0] \\
(0.4+x) / 0.2, \quad x \in[-0.4,-0.2)
\end{array} \quad \mu_{i}^{M D}=\left\{\begin{array}{l}
(-0.3-x) / 0.2, \quad x \in[-0.5,-0.3] \\
(x+0.7) / 0.2, \quad x \in[-0.7,-0.5)
\end{array}\right.\right. \\
& \mu_{i}^{V D}=\left\{\begin{array}{l}
(-0.6-x) / 0.2, \quad x \in[-0.8,-0.6] \\
1, \quad x \in[-1,-0.8)
\end{array}\right.
\end{aligned}
$$

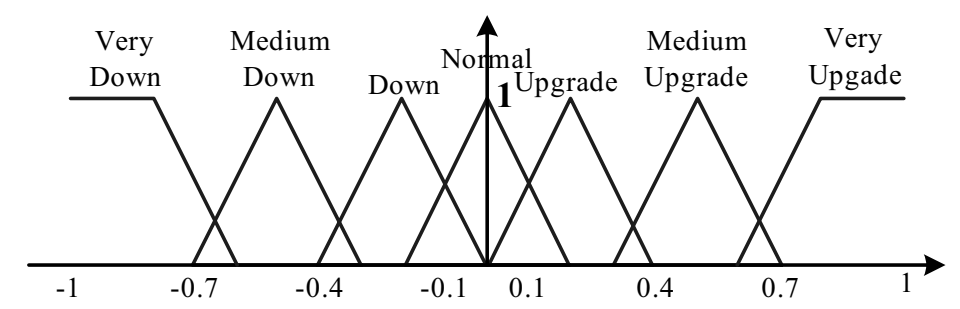

Figure 1. Fuzzifying the temporal variable

\subsection{Weighted Medical Rules}

To implement the uncertain medical inference and diagnosis, besides the preceding methodology of temporal fuzzy variables, most of the medical rules are also assessed via the certainty factors as well (Giarratano, 1998). Consequently, the classical medical rules could be described as following: 


$$
R_{t}:\left\{\wedge\left[(\text { Sign, Attribute })_{i} \mid \text { Hypothesis }_{i}\right]\right\} \Rightarrow\left\{\wedge(\text { Disease } \mid \text { Hypothesis })_{j}\right\} C F_{t}
$$

where the subscript ' $t$ ' means the $t$-th medical rule; ' $i$ ' is the $i$-th antecedent and similarly, ' $j$ ' is the $j$-th consequent of the specific medical rule. Moreover, "Sign" belongs to the collected vital signs for health assessing and its attribute is the preceding fuzzy variable such as "Very Upgrade", "Medium Upgrade", etc. And the symbol '^' stands for the logic " $A N D$ " while 'I' means the alternative item. Finally, " $C F$ " is the certainty factor grading the specific medical rule.

As to the medical diagnosis, in general, it commences with the peripheral factors such as medical history, appearance symptoms and so on. Hereby medical professionals could derive several possible diseases from the known medical information. Then, the final diagnosis will only be concluded from the object-oriented measuring or testing. Consequently, in terms of the medical rules, the symptoms or signs in antecedents play the different contributive roles for the consequents. To improve the uncertain diagnosis, it is natural to weight the antecedents of medical rules.

Here the methodology based on statistical average and generalization is proposed to weight the antecedents. For the specific $i$-th antecedent " (Sign, Attribute)," or "Hypothesis", if it exists in $n$ piece of medical rules, the corresponding weight could be worked out as follows.

$$
\text { weight }_{i}=\left(\sum_{k=1}^{n} C F_{n k}\right) / n
$$

Then, for each of medical rule, the weights of antecedents could be further generalized as (8). For example, if the $t$-th medical rule comprises $p$ antecedents, the $k$-th weight:

$$
\text { weight }_{t k}^{*}=\text { weight }_{t k} / \sum_{w=1}^{p} \text { weight }_{t w} \quad(k \in[1, p])
$$

Finally, the mentioned rules as (6) will be converted into the medical rules compliant to (9):

$$
R_{t}:\left\{\wedge\left[(\text { Sign }, \text { Attribute })_{i} \mid \text { Hypothesis }_{i}, \text { weight }_{i}\right]\right\} \Rightarrow\left\{\wedge(\text { Disease } \mid \text { Hypothesis })_{j}\right\} C F_{t}
$$

\section{Embedded Medical Advisory Systems}

As mentioned previously, the embedded-link, self-adaptive medical advisory system will be integrated with the medical sensors for pervasive health monitoring. Here the "embedded-link" system means it should be well designed and ready for the embedded platforms of mobile or wearable 
medical sensors. Linking to central server enables it to provide the effective health services. And the "self-adaptive" system refers to running independently for the various medical sensors and home users due to the customized and updatable diagnostic knowledge base.

\subsection{Accompanied Knowledge Base}

Since the module of machine learning for medical knowledge discovery is generally built on the tremendous dataset and moreover, depends on the support of medical experts, it is unfeasible and also not necessary to implement the complicated module in the embedded platform. The embedded medical advisory system hereby comprises the module of inference machine and a customized \& updatable knowledge base. Here "customized" refers to only putting those essential medical rules related to the specific medical sensor and health condition in local knowledge base. Via up linking to the global comprehensive knowledge base residing in health centers, each of the individual embedded medical advisory system could be calibrated conveniently while necessary.

During the initialization, the relevant medical information is firstly submitted to health centers. Assume that the submitted information could be formatted as " $\left\{\wedge(\text { Entity, Value } \mid \text { Attribute })_{q}\right\}$ ", where the "Entity" belongs to either the collected vital signs or the basic physiological \& physical parameters of home user. Moreover, as mentioned previously, the medical rules in global knowledge base are compliant to (9). Therefore, to customize the local knowledge base is to search \& match the most suitable medical rules according to the submitted information, and then download them to the local accompanied knowledge bases. This customizing and updating procedure can be conducted dynamically during system initialization or data backup.

\subsection{Inference Machine}

Given the uncertain medical rules with temporal fuzzy variables, the classical fuzzy inference is built on the "conjunction-disjunction" operation (Yager, 1992). Now assume that the final diagnosis could be specified as "CR (Disease $e_{j}$ )", where "CR" is the acronym of "Confidence of Result" corresponding to the "CF: Certainty Factor". Then, for the medical rule compliant to (6), the fuzzy inference formula could be summarized as (10): 


$$
C R\left(\text { Disease }_{j}\right)=\left\{\vee\left[C F_{t} *\left\{\wedge \mu\left[\left(\text { Sign, } \text { Atribute }_{i} \mid \text { Hypothesis }_{i}\right)\right]_{t}\right\}\right]\right\}
$$

where ' $\vee$ ' is the operator of "disjunction" and " $\wedge$ ' is "conjunction".

However, due to weighted, here the uncertainty comes from not only the fuzzy variables but also the weights of medical rules. Consequently, in the proposed embedded medical advisory system, the inference machine should be modified accordingly. Similarly, the first is also an accumulative procedure because of the incomplete matching. In (11), the involved "hypotheses"should be firstly worked out for following medical diagnosis. Then the modified formula in (12) acts as the inference foundation of medical advisory system.

$$
\begin{gathered}
C R\left(\text { Hypothesis }_{l}\right)=\left\{\vee\left[C F_{m} * \sum_{p} \mu\left(v s_{m p}\right) * \text { weight }_{m p}\right]\right\} \\
C R\left(\text { Disease }_{k}\right)=\left\{\vee\left[C F_{n} * \sum_{q} \mu\left(v s_{n q}\right) *\left(\text { weight }_{n q} *\left[1 \mid C R_{l}\right]\right)\right]\right\}
\end{gathered}
$$

\subsection{Shell}

In the knowledge-based system, "shell" means that the inference machine is separated from the knowledge base so to run adaptively for the different applications. Here due to the limited systematic resources, as mentioned previously, there are only the customized medical rules in the accompanied knowledge base. Naturally, the embedded medical advisory system needs to update its knowledge base while necessary. Benefited from the distributed framework of network communication, the local knowledge base is convenient to be updated and modified.

Eventually, to implement the pervasive health monitoring, the accompanied knowledge base should be dynamically updated so that the embedded medical advisory system keeps effective. For example, once the collected symptoms are out of the boundary of knowledge base, the embedded medical advisory system will report the related information to health centers. If it is under the control of global medical knowledge base, the inference results accompanied with the updated knowledge base will be fed back accordingly. Otherwise, the request will be transferred to medical professionals. Not only the medical diagnosis is sent back, but also the accompanied medical knowledge base will be reviewed and revised by medical professionals. Moreover, due to the ongoing machine learning for medical knowledge discovery, the embedded medical advisory system will be regularly calibrated too. 


\section{Prototyping System}

Presently, the proposed medical advisory system has been incorporated into a mobile cardiovascular monitoring system based on the hemodynamic analysis of sphygmogram. It firstly works out a group of vital signs from the sampled sphygmogram, which reflects the health profile of cardiovascular system including the cardiac and microcirculation function, vascular and blood status, etc. Therefore, besides conserved for later reference, these vital signs are also submitted to the embedded medical advisory system. In virtue of the proposed embedded-link selfadaptive medical advisory system, if the effective medical advices are provided, they will be fed back to home users directly. Otherwise, the collected medical information will be submitted to health center and asks for the medical assistance.

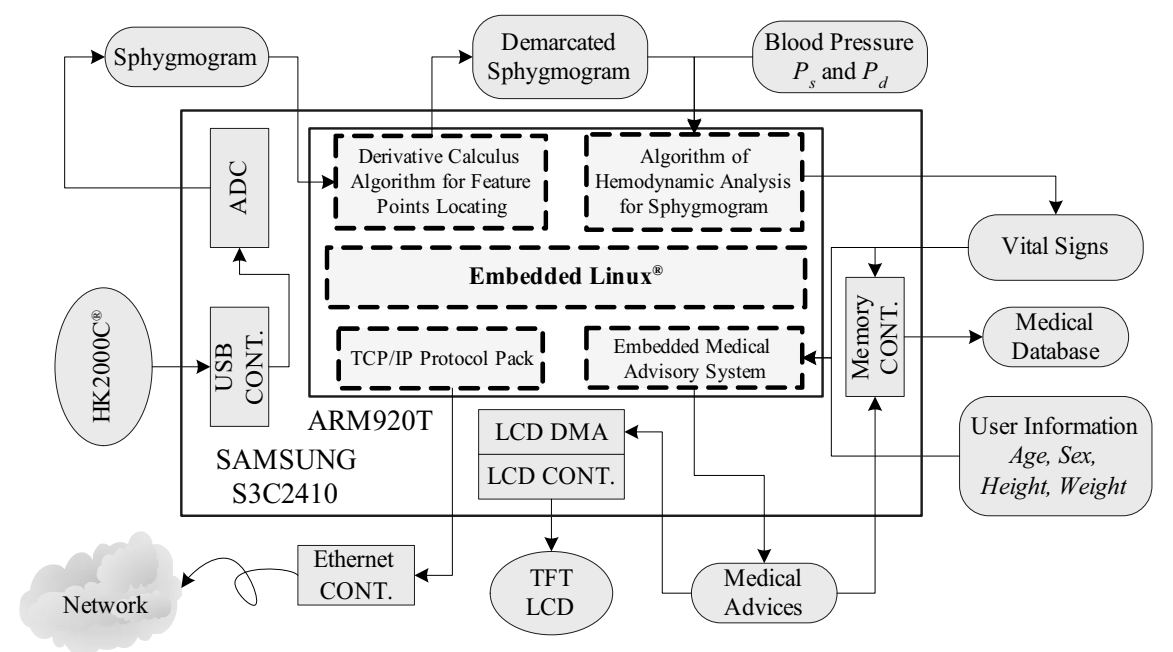

Figure 2. The ARM-based intelligent mobile cardiovascular monitoring system

Here the medical sensor - "HK2000C " " By Hefei Huake Electron Graduate School, China) -is adopted for sampling of sphygmogram. The collected data are then submitted to a self-contained developing platform of embedded systems -"HHARM2410 "( By HHTech Co. Ltd., China) via the integrated USB port. The following signal processing and data analyzing, coordinated by the on-system "embedded Linux ${ }^{\otimes}$," is condensed into a micro system, shown in Figure 3, with the sophisticated ARM9 chip "Samsung ${ }^{\circledR}$ S3C2410", 64M SDRAM and 16M Flash ROM, etc. Then, with the extended peripheral interfaces such as "Ethernet interface" and "TFT LCD", the mobile device with embedded medical advisory system 
could intelligently monitor and analyze the health condition of cardiovascular system via sphygmogram.

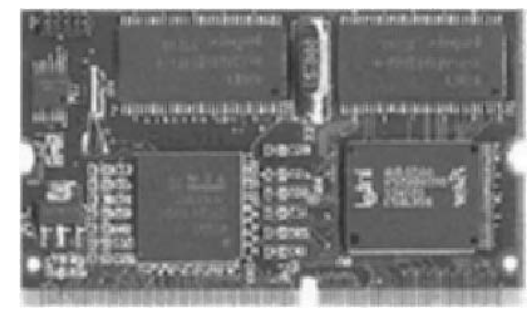

Figure 3. The core module of prototyping system

\section{Conclusion}

In this paper, an embedded-link, self-adaptive medical advisory system is proposed for pervasive health monitoring. The system is well designed and implemented for the embedded platform of mobile or wearable medical sensors. Moreover, to conduct the uncertainty medical diagnosis, the methodologies of soft computing, such as temporal fuzzy variables and weighted medical rules, are applied to the proposed medical advisory system. Finally, it is implemented and verified in a mobile cardiovascular monitoring system.

In the future, the proposed system could be further enhanced. For example, though the weighted medical rules with temporal fuzzy variables are effective to deal with the uncertainty medical diagnosis, the newer methodologies of soft computing such as fuzzy neural networks will be more competitive for the embedded systems. And it is also attractive to implement the proposed system for the autonomous interpreter of electrocardiogram.

\section{Acknowledgments}

The authors would like to acknowledge the financial support of Research Committee of University of Macau (Grant No: 3676) in our research project 'Network-based, Intelligent, Home Healthcare System'. 


\section{References}

D. Castro, J. Presedo, M. Fernandez-Delgado, and S. Barro (2001) Patient telemonitoring at home. In: Proceedings of $23^{\text {rd }}$ Annual EMBS International Conference, pp. 3571-3574.

Diana M., Radu M., Wungmee P., and Sundaresan J. (2003) Ready to Ware. IEEE Spectrum, pp. 28-32.

D. Franchi, A. Belardinelli, G. Palagi, A. Ripoli, and R. Bedini (1998) New telemedicine approach to the dynamic ECG and other biological signals ambulatory monitoring. Computers in Cardiology 25: 213-215.

G. C. Crumley, N. E. Evans, W. G. Scanlon, J. B. Burns, and T. G. Trouton (2000) The design and performance of a $2.5-\mathrm{GHz}$ telecommand link for wireless biomedical monitoring. IEEE Trans. Inform. Technol. Biomed. 4(4): 285-291.

J. Giarratano, and G. Riley (1998) Inexact reasoning. In: Expert Systems Principles and Programming. PWS Publishing Company, pp. 227-308.

M. Nambu, K. Nakajima, A. Kawarada, and T. Tamura (2000) A system to monitor elderly people remotely using the power line network. In: Proceedings of $22^{\text {nd }}$ Annual EMBS International Conference (1): 782-785.

M. Ogawa, R. Suzuki, and S. Otake (2002) Long term remote behavioral monitoring of elderly by using sensors installed in ordinary house. In: Proceedings of $2^{\text {nd }}$ Annual International IEEE-EMBS Special Topic Conference on Microtechnologies, Medical and Biology, pp. 322-325.

R.R. Yager, and L.A. Zadeh (1992) Expert systems using fuzzy logic. In: An Introduction to Fuzzy Logic Applications in Intelligent Systems. Kluwer Academic Publishers, Norwell, Massachusetts.

T. C. Jannett, S. Prashanth, S. Mishra, and V. Ved (2002) An intelligent telemedicine system for remote spirometric monitoring. In: Proceedings of $34^{\text {th }}$ Southeastern Symposium on System Theory, pp. 53-56.

W. Pedrycz, and F. Gomide (1998) Linguistic Variables. In: An Introduction to Fuzzy Sets. The MIT Press, Cambridge, Massachusetts. 